\title{
Postoperative Management of Eosinophilic Chronic Rhinosinusitis with Nasal Polyps: Impact of High-Dose Corticosteroid Nasal Spray
}

\author{
Katsuhisa Ikeda ${ }^{1}$ Shin Ito ${ }^{1}$ Remi Hibiya ${ }^{1}$ Hirotomo Homma ${ }^{1}$ Noritsugu Ono ${ }^{1}$ Hiroko Okada ${ }^{1}$ \\ Yoshinobu Kidokoro ${ }^{1}$ Akihito Shiozawa ${ }^{1}$ Takeshi Kusunoki ${ }^{1}$ \\ ${ }^{1}$ Department of Otorhinolaryngology, Juntendo University, Tokyo, Japan \\ Int Arch Otorhinolaryngol 2019;23:101-103.

\begin{abstract}
Address for correspondence Katsuhisa Ikeda, MD, Department of Otorhinolaryngology, Juntendo University, 2-1-1 Hongo, Bunkyo-ku,
\end{abstract} \\ Tokyo 113-8421, Japan (e-mail: ike@juntendo.ac.jp).
}

\begin{abstract}
Keywords

- chronic rhinosinusitis nasal polyps

- adverse effects

- intranasal administration

- corticosteroids

Introduction Eosinophilic chronic rhinosinusitis (ECRS) is characterized by an eosinophilic inflammation driven by Th2-type cytokines. Glucocorticosteroids are the most common first-line treatment for ECRS with nasal polyps.

Objective We have evaluated the long-term treatment with double-dose intranasal corticosteroids in refractory ECRS nasal polyps resistant to the conventional dose and assessed the risk of adverse systemic effects

Methods Sixteen subjects were enrolled in this study. All subjects had ECRS after endoscopic sinus surgery that resulted in recurrent mild and moderate nasal polyps and were undergoing a postoperative follow-up application of mometasone furoate at a dose of 2 sprays $(100 \mu \mathrm{g})$ in each nostril once a day $(200 \mu \mathrm{g})$. All the patients were prescribed mometasone furoate, administered at a dose of 2 sprays $(100 \mu \mathrm{g})$ in each nostril twice a day $(400 \mu \mathrm{g})$ for 6 months.

Results The average scores of the symptoms during the regular dose of intranasal steroid treatment were $5.2 \pm 2.2$, but 6 months after the high-dose application, they had significantly decreased to $2.5 \pm 1.4(p<0.05)$. The polyp size showed an average score of 1.38 during the regular dose which was significantly reduced to 0.43 $(p<0.01)$ by the double dose. Glycated hemoglobin (HbA1c) showed normal ranges in all the patients tested. The cortisol plasma concentration was also normal.

Conclusion Doubling the dose of the nasal topical spray mometasone furoate might be recommended for the treatment of recurrent nasal polyps in the postoperative follow-up of intractable ECRS.
\end{abstract}

\section{Introduction}

Chronic rhinosinusitis (CRS) is defined as persistent inflammation of the nasal and paranasal cavity mucosa lasting $\geq 3$ months ${ }^{1}$. Based on an epidemiological study in the United States, $\sim 29.2$ million adults (a prevalence of $14.2 \%$ ) have CRS. The prevalence and medical costs of CRS are increasing and have become important social issues. ${ }^{2}$ The histomorphological patterns of CRS with nasal polyps are characterized by the predominance of eosinophils and mixed mononuclear

received

February 7, 2018

accepted

May 26, 2018

published online

October 24, 2018 10.1055/s-0038-1668515. ISSN $1809-9777$. cells but a relative paucity of neutrophils, ${ }^{3}$ and therefore can be designated as eosinophilic CRS (ECRS). Mucosal infiltration with eosinophils in CRS with nasal polyps may be more refractory to surgical cure and is frequently associated with bronchial asthma. ${ }^{4}$

Eosinophilic CRS is characterized by an eosinophilic inflammation driven by Th2-type cytokines. ${ }^{5}$ Since glucocorticosteroids have potent anti-inflammatory effects that include the decrease of the inflammation mediated by eosinophils, ${ }^{6,7}$ they are the most common first-line

Copyright $\odot 2019$ by Thieme Revinter Publicações Ltda, Rio de Janeiro, Brazil

\section{License terms}

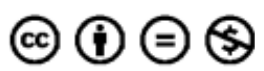


treatment for CRS with nasal polyps. ${ }^{8}$ Placebo-controlled studies have shown that topical corticosteroid therapy reduces the recurrence of polyps after surgery. ${ }^{9,10}$ However, since topical corticosteroid therapy is not effective in all patients, systemic glucocorticosteroids are sometimes used. One placebo-controlled trial demonstrated the efficacy of a short-term course of oral corticosteroids for improving symptoms and reducing polyp size. ${ }^{11}$ Although short courses of oral corticosteroids are safe in CRS with nasal polyps, ${ }^{8}$ repeated or prolonged use of oral steroids may be associated with an enhanced risk of systemic side effects. ${ }^{12}$

In the present study, we have evaluated the long-term treatment with double-dose intranasal corticosteroids in refractory ECRS nasal polyps resistant to the conventional dose and assessed the risk of adverse systemic effects such as hypothalamic-pituitary-adrenal axis suppression and sugar tolerance.

\section{Method}

The diagnosis of eosinophilic CRS was diagnosed based on the criteria of the Japanese Epidemiological Survey of Refractory Eosinophilic Chronic Rhinosinusitis (JESREC) study. ${ }^{13}$ Patients with specific types of CRS with nasal polyps including aspirin sensitivity and cystic fibrosis were excluded. Endoscopic sinus surgery was basically performed according to our previous paper. ${ }^{14}$ Surgery in all cases was performed under general anesthesia. After postoperatively ineffective, prolonged application of mometasone furoate at a dose of 2 sprays $(100 \mu \mathrm{g})$ in each nostril once a day $(200 \mu \mathrm{g})$, recurrent mild and moderate nasal polyps were prospectively entered into the clinical study. Sixteen subjects with eosinophilic CRS ( 3 female and 13 male, ranging in age from 37 to 63 years old, with a mean age of 49 years old) admitted to the department of otorhinolaryngology of the outpatient clinic of the Juntendo University Faculty of Medicine between January 2010 and July 2014 were enrolled. All the patients were administered mometasone furoate at a dose of 2 sprays $(100 \mu \mathrm{g})$ in each nostril twice a day $(400 \mu \mathrm{g})$ for 6 months. Concomitant medications that were not allowed during the study included oral corticosteroids (excluding inhaled corticosteroid for mild-to-moderate persistent asthma), antihistamines, decongestants, and leukotriene pathway modifiers. The presence of nasal discharge, postnasal drainage, nasal obstruction, headache and anosmia were recorded and their severity was defined as follows: severe $=3$, moderate $=2$, slight $=1$ and absent $=0$ based on a previous paper. ${ }^{14}$ Polyp size was graded for each nasal cavity on a scale of 0 to $3(0$, no polyp; 1 , polyps in the middle meatus; 2 , polyps reaching below the inferior border of the middle turbinate but not the inferior border of the inferior turbinate; 3 , large polyps reaching to or below the inferior border of the inferior turbinate or polyps medial to the middle turbinate. ${ }^{9}$ At the end of the study, glycated hemoglobin (HbA1c) and cortisol plasma concentration were analyzed. The study was approved by the ethics committee of the Juntendo University Faculty of Medicine. All subjects entered the study after signing an informed consent form.

The data were expressed as the mean \pm standard deviation (SD) Statistical analyses were evaluated using paired-t test in StatMate IV for Windows (GraphPad Software, La Jolla, CA, USA). Differences were considered to be significant if $p<0.05$.

\section{Results}

The average scores of the symptoms during the regular dose of intranasal steroid treatment were $5.2 \pm 2.2$, but 6 months after high-dose application, they had significantly decreased to $2.5 \pm 1.4(p<0.05)$. Specifically, all the patients had recovered from severe $(n=9)$ and moderate $(n=7)$ anosmia to slight anosmia $(n=10)$ and normal olfaction $(n=6)$. Polyp size changed from a score of 2 to 0 in one patient, from a score of 1 to 0 in 8 patients, from a score of 2 to 1 in 5 patients, and from a score of 1 to 1 in 2 patients. Polyp size showed an average score of 1.38 during the regular dose and was significantly reduced to a score of $0.43(P<0.01)$ after doubling the dose for a period of 6 months.

In all the patients tested, the HbA1c ranges were normal. The cortisol plasma concentrations were also within the normal range (-Fig. $\mathbf{1}$ ).

\section{Discussion}

The present study revealed that a high-dose of the corticosteroid nasal spray mometasone furoate significantly improved nasal symptoms including smell acuity and reduced polyp size, without long-term side effects, compared with the regular dose. It is well known that nasal blockage and rhinorrhea respond well to topical nasal steroids, whereas controversial findings on the improvement of the sense of smell have been reported. ${ }^{15,16}$ The sense of smell constitutes an important warning system for gas leaking, smoke, food spoilage, and air pollution. Eosinophilic CRS is a major cause of smell loss, having an important impact on the quality of life of the patients and constituting an especially troublesome symptom in the elderly that is associated with the loss of appetite and weight, and the onset of depression.

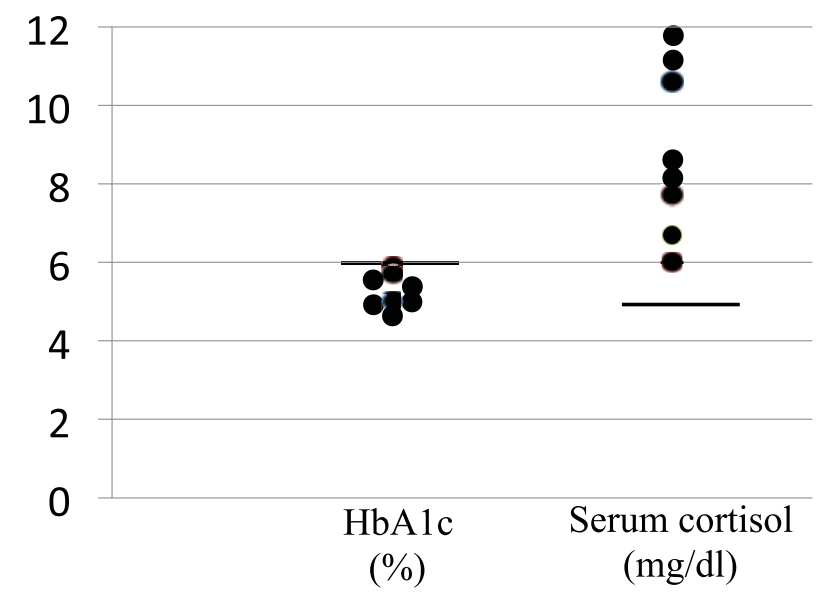

Fig. 1 Distribution of glycated hemoglobin (HbA1c) and cortisol serum concentrations after doubling the dose the nasal topical spray mometasone furoate. The bars show the upper and lower normal ranges in $\mathrm{HbA} 1 \mathrm{c}$ and cortisol serum concentrations, respectively. 
The dose-response effects for fluticasone in asthma were reported ${ }^{17}$ and are likely compatible with those of the upper airway inflammation.

Although nasal topical corticosteroids are very safe in general, several factors such as the molecular properties of corticosteroids, dose prescription, mode of delivery, and severity of the underlying disease may influence steroid absorption into the systemic circulation. ${ }^{18}$ The systemic bioavailability of intranasal corticosteroids varies from some new molecules to older molecules, which may influence the risk of adverse systemic effects. ${ }^{19}$ There is no clear evidence of a correlation between the use of nasal corticosteroids at the recommended doses and systemic changes in bone mineral biology, cataracts, or glaucoma. At the recommended doses, adrenal suppression may occur with some nasal corticosteroids but the clinical relevance remains uncertain, whereas the overuse of nasal corticosteroids may be responsible for adrenal insufficiency and a decrease in bone mineral density. ${ }^{20}$ Mometasone furoate applied to the skin has been demonstrated in clinical studies to have a low potential of causing systemic side effects including hypothalamic-pituitary-adrenal axis suppression. ${ }^{21,22}$ An aqueous nasal spray formulation of mometasone furoate has been developed for the treatment of allergic rhinitis, based upon the prediction that this corticosteroid would be devoid of systemic activity when applied in the nose. Mometasone furoate applied to the nasal mucosa at doses up to 20 times the common clinical dose demonstrated no effect on the plasma cortisol area under the curve, urinary free cortisol, or morning plasma cortisol level. ${ }^{23}$ Thus, a nasal steroid spray of high-dose corticosteroid may result in beneficial effects for intractable ECRS without adverse systemic effects.

\section{Conclusion}

Doubling the dose of the nasal topical spray mometasone furoate might be recommended for the treatment of recurrent nasal polyps in the postoperative follow-up of intractable ECRS.

\section{References}

1 Benninger MS, Ferguson BJ, Hadley JA, et al. Adult chronic rhinosinusitis: definitions, diagnosis, epidemiology, and pathophysiology. Otolaryngol Head Neck Surg 2003;129(3, Suppl): $\mathrm{S} 1-\mathrm{S} 32$

2 Kaliner MA, Osguthorpe JD, Fireman P, et al. Sinusitis: bench to bedside. Current findings, future directions. J Allergy Clin Immunol 1997;99(6 Pt 3):S829-S848

3 Hamilos DL, Leung DYM, Wood R, et al. Chronic hyperplastic sinusitis: association of tissue eosinophilia with mRNA expression of granulocyte-macrophage colony-stimulating factor and interleukin-3. J Allergy Clin Immunol 1993;92(1 Pt 1):39-48

4 Dinis PB, Gomes A. Sinusitis and asthma: how do they interrelate in sinus surgery? Am J Rhinol 1997;11(06):421-428
5 Van Zele T, Claeys S, Gevaert P, et al. Differentiation of chronic sinus diseases by measurement of inflammatory mediators. Allergy 2006;61(11):1280-1289

6 Mygind N, Lildholdt T. Nasal polyps treatment: medical management. Allergy Asthma Proc 1996;17(05):275-282

7 Badia L, Lund V. Topical corticosteroids in nasal polyposis. Drugs 2001;61(05):573-578

8 Fokkens W, Lund V, Mullol J; European Position Paper on Rhinosinusitis and Nasal Polyps Group. EP3OS 2007: European position paper on rhinosinusitis and nasal polyps 2007. A summary for otorhinolaryngologists. Rhinology 2007;45(2, suppl. 20):97-101

9 Stjärne P, Olsson P, Alenius M. Use of mometasone furoate to prevent polyp relapse after endoscopic sinus surgery. Arch Otolaryngol Head Neck Surg 2009;135(03):296-302

10 Dingsør G, Kramer J, Olsholt R, Søderstrøm T. Flunisolide nasal spray $0.025 \%$ in the prophylactic treatment of nasal polyposis after polypectomy. A randomized, double blind, parallel, placebo controlled study. Rhinology 1985;23(01):49-58

11 Hissaria P, Smith W, Wormald PJ, et al. Short course of systemic corticosteroids in sinonasal polyposis: a double-blind, randomized, placebo-controlled trial with evaluation of outcome measures. J Allergy Clin Immunol 2006;118(01):128-133

12 Walsh LJ, Lewis SA, Wong CA, et al. The impact of oral corticosteroid use on bone mineral density and vertebral fracture. Am J Respir Crit Care Med 2002;166(05):691-695

13 Tokunaga T, Sakashita M, Haruna T, et al. Novel scoring system and algorithm for classifying chronic rhinosinusitis: the JESREC Study. Allergy 2015;70(08):995-1003

14 Ikeda K, Kondo Y, Sunose H, et al. Subjective and objective evaluation in endoscopic sinus surgery. Am J Rhinol 1996; 10:217-220

15 Benítez P, Alobid I, de Haro J, et al. A short course of oral prednisone followed by intranasal budesonide is an effective treatment of severe nasal polyps. Laryngoscope 2006;116(05): 770-775

16 Stjärne P, Blomgren K, Cayé-Thomasen P, Salo S, Søderstrøm T. The efficacy and safety of once-daily mometasone furoate nasal spray in nasal polyposis: a randomized, double-blind, placebo-controlled study. Acta Otolaryngol 2006;126(06):606-612

17 Powell H, Gibson PG. Inhaled corticosteroid doses in asthma: an evidence-based approach. Med J Aust 2003;178(05):223-225

18 Cave A, Arlett P, Lee E. Inhaled and nasal corticosteroids: factors affecting the risks of systemic adverse effects. Pharmacol Ther 1999;83(03):153-179

19 Holm AF, Fokkens WJ, Godthelp T, Mulder PG, Vroom TM, Rijntjes E. A 1-year placebo-controlled study of intranasal fluticasone propionate aqueous nasal spray in patients with perennial allergic rhinitis: a safety and biopsy study. Clin Otolaryngol Allied Sci 1998;23(01):69-73

20 Licata AA. Systemic effects of fluticasone nasal spray: report of 2 cases. Endocr Pract 2005;11(03):194-196

21 Vernon HJ, Lane AT, Weston W. Comparison of mometasone furoate $0.1 \%$ cream and hydrocortisone $1.0 \%$ cream in the treatment of childhood atopic dermatitis. J Am Acad Dermatol 1991;24 (04):603-607

22 Mösges R, Bachert C, Rudack C, et al. Efficacy and safety of mometasone furoate nasal spray in the treatment of chronic rhinosinusitis. Adv Ther 2011;28(03):238-249

23 Bruni FM, De Luca G, Venturoli V, Boner AL. Intranasal corticosteroids and adrenal suppression. Neuroimmunomodulation 2009;16(05):353-362 